\title{
Diagnóstico laboratorial de raiva em quirópteros realizado em área metropolitana na região sudeste do Brasil
}

\section{Laboratory diagnosis of rabies in chiroptera carried out of a metropolitan area of South- eastern Brazil}

\author{
Marilene F. Almeida*, Elizabeth A. C. Aguiar*, Luiza F. A. Martorelli”, Miriam M. S. Silva*
}

\begin{abstract}
ALMEIDA, M. F. et al. Diagnóstico laboratorial de raiva em quirópteros realizado em área metropolitana na região sudeste do Brasil. Rev. Saúde Pública, 28: 341-4, 1994. No período de janeiro de 1988 a dezembro de 1992, foi realizado diagnóstico de raiva em 289 morcegos através das técnicas de imunofluorescência direta e de inoculação intracerebral em camundongos. Dois morcegos insetívoros da espécie Nyctinomops macrotis se apresentaram positivos, representando 0,69\% da amostra. Esses morcegos foram capturados, ainda vivos, em 1988 e 1990, na sala de um apartamento no sétimo andar e no muro de uma casa, respectivamente. Ambos em bairros residenciais. Apresentaram período de incubação de 13 e ll dias, respectivamente, na prova biológica. A existência de morcegos insetívoros infectados com o vírus da raiva é preocupante, uma vez que essa população parece ser cada vez mais freqüente em áreas urbanas, porém isto não justifica ações predatórias indiscriminadas contra as espécies, principalmente levando-se em consideração a importância do morcego no equilibrio ecológico da população de insetos, abundante em área urbana.
\end{abstract}

Descritores: Quirópteros, microbiologia. Vírus da raiva, isolamento. Raiva, diagnóstico.

\section{Introdução}

Na Saúde Pública, o morcego tem sido estudado como possivel reservatório natural e/ou transmissor de agentes patogênicos, entre eles ,o vírus rábico. Essa hipótese foi levantada pela primeira vez por Carini ${ }^{4}$ em 1911, ao estudar uma epizootia em Santa Catarina, e foi confirmada em 1925 por Haupt e Rehaag ${ }^{11}$ com o isolamento do vírus rábico de material nervoso do morcego Phyllostoma superciliatum. O vírus foi isolado pela primeira vez em morcegos insetívoros por Pawan ${ }^{14}$, em 1936, na Ilha de Trinidad, onde no período de 1925 a 1936, foram registrados 89 óbitos humanos pela doença ${ }^{1,5}$.

Nos EUA, a presença do vírus rábico em morcegos insetívoros (Dasypterus floridanus e Lasiurus seminolus) foi demonstrada, pela primeira vez, por Venters e col. ${ }^{21}$ em 1953, na Flórida. Desde então, mais de 30 espécies infectadas pelo vírus da raiva foram identificadas, entre as 39 espécies que vivem no norte do México, EUA e Canadás. Registrando-se, inclusive, 10 mortes humanas, creditadas à exposição a morcegos $^{6,10}$.

* Centro de Controle de Zoonoses da Prefeitura do Município de São Paulo

Separatas/Reprints: M. F. Almeida - Rua Santa Eulália, 86 02031-020 - São Paulo, SP - Brasil

Edição subvencionada pela FAPESP. Processo 94/0500-0.
Na Europa, em 1954, foi relatado um caso de raiva transmitida por morcego em Hamburgo, Alemanha, porém nos 30 anos seguintes, foram registrados apenas casos esporádicos e geograficamente bem distantes entre $\mathrm{si}^{8}$.

Na década de 80 , os morcegos voltaram a chamar a atenção com a ocorrência de uma epizootia na Europa $^{8}$. Em 1983 uma criança morreu na antiga URSS, 21 dias após uma mordida no lábio e em 1985 um pesquisador finlandês morreu de raiva após ser repetidamente mordido por morcegos.

Por essa razão, alguns países da Europa preocuparam-se em investigar a presença do vírus rábico na população de quirópteros ${ }^{8}$. Em 1986, a Dinamarca analisou 550 morcegos, dos quais 104 apresentaram-se positivos. Todos, menos dois, eraminsetívoros da espécie $E$. serotinus. A Holanda examinou 1.250 morcegos, dos quais 86 apresentaram-se positivos ( 83 $E$. serotinus e 3 M.dasycneme). O Reino Unido e a Suíça analisaram 126 e 117 morcegos, respectivamente, durante 1987, e todos foram negativos. A Inglaterra examinou 500 morcegos no período de janeiro de 1985 a outubro de 1988, encontrando 9 positivos.

No Brasil, em 1961, Silva e col. ${ }^{16}$ isolou o vírus do morcego Phyllostomus hastatus hastatus, o mesmo ocorrendo em 1965, no Rio Grande do Sul quando Bauer e Crusius ${ }^{3}$ isolaram o vírus do morcego insetívoro Tadarida brasiliensis. 
Desde então, 24 espécies de morcegos já foram diagnosticadas com raiva no Brasil, 14 da família Phyllostomidae, 6 da família Molossidae e 4 da família Vespertilionidae ${ }^{19}$.

No Município de São Paulo, o Centro de Controle de Zoonoses mantém um serviço de diagnóstico de raiva em morcegos, através do qual foi possível detectar em 1988 e 1990, 2 morcegos insetívoros comprovadamente infectados com o vírus da raiva. Ambos foram classificados como Nyctinomops macroti ${ }^{20}$. A partir do primeiro diagnóstico positivo, foi ampliado o serviço de orientação ao público, acionando, quando necessário, o serviço de captura, e realizando o diagnóstico e a classificação de todos os morcegos enviados.

$O$ presente trabalho objetiva contribuir para o conhecimento da epidemiologia da raiva em morcegos de área urbana pela apresentação dos resultados da pesquisa do vírus e das familias e seus hábitos alimentares, realizada no período de janeiro de 1988 a dezembro de 1992, e analisar o comportamento do vírus rábico nessas espécies.

\section{Material e Método}

Foram retirados de morcegos encaminhados ou capturados, vivos, mortos ou congelados, o cérebro, as glândulas salivares e a gordura interescapular.

O conjugado anti-rábico fluorescente utilizado com título 1:40 foi elaborado pela técnica de cromatografia e Deae celulose ${ }^{12}$.

Os camundongos suiços albinos utilizados eram procedentes de biotério próprio, com 21 dias e 11 a 14 gramas.

O vírus C.V.S. $31 / 2$ utilizado era procedente do INPPAZ-Instituto Panamericado de Proteção de Alimentos e Zoonoses.

A classificação seguiu a chave para determinação de quirópteros brasileiros de Vizotto e Taddei ${ }^{22}$.

O diagnóstico foi feito pela detecção da presença do antígeno rábico no cérebro do morcego, de acordo com a técnica de imunofluorescência direta(IFD) ${ }^{12}$ e técnica de inoculação intracerebral em camundongo (IICC) ${ }^{23}$.

A partir de 1991, além do cérebro, também foram processados pela técnica de IICC uma suspensão das glândulas salivares e da gordura interescapular, e o período de observação foi ampliado de 21 para 42 dias.

\section{Resultados}

Dos 301 morcegos recebidos ou capturados no período de 1988-1992, 12 foram desprezados por conservação inadequada (formol, éter ou álcool) e

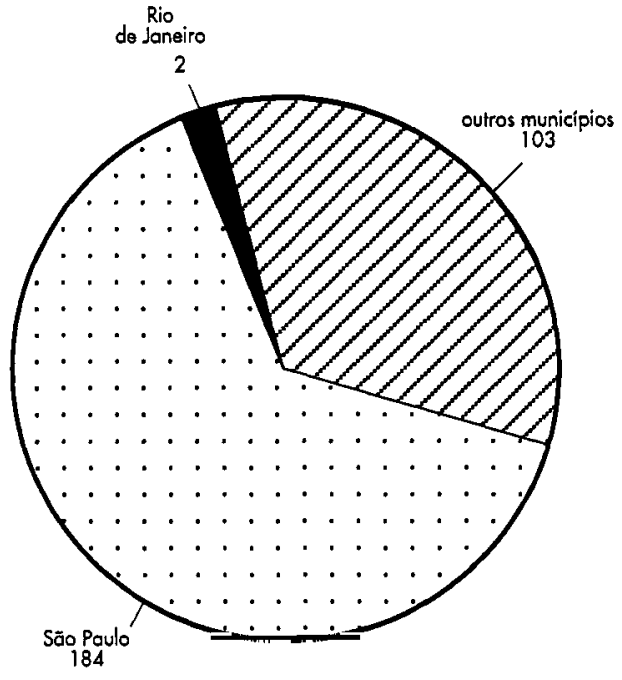

Figura 1: Espécimes de quirópteros recebidos para diagnóstico de raiva, segundo procedência, período 1988 a 1992.

autólise, 289 foram submetidos ao diagnóstico de raiva. Destes, 184 eram procedentes do Município de São Paulo, 103 eram do interior do Estado de São Paulo e 2 do Rio de Janeiro (Fig. 1).

Durante esse período observou-se uma tendência de aumento no número de espécimes recebidos para análise (Fig. 2).

Dos 289 morcegos diagnosticados pelas provas de IFD e IICC, 287 se apresentaram negativos e 2 positivos, que representam $0,69 \%$ da amostra.

Os 2 morcegos positivos ocorreram nos anos de 1988 e 1990 e foram classificados como Nyctinomops macrotis. No primeiro caso não foi possível saber o sexo e no segundo tratava-se de uma fêmea adulta. Os dois foram capturados em bairros residenciais do Município de São Paulo.O primeiro apresentou período de incubação de 13 dias e o segundo de 11 dias no teste IICC.

Os testes de IICC realizados com a glândula salivar e gordura interescapular, nos 160 morcegos

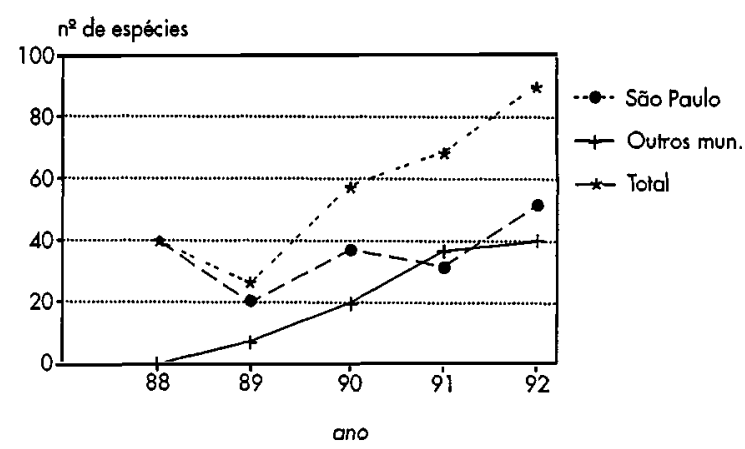

Figura 2: Distribuição dos quirópteros, segundo procedência no período 1988 a 1992. 
de 1991 e 1992, resultaram todas negativas. Neste teste todos os camundongos infectados foram observados diariamente por 42 dias e não apresentaram nenhuma sintomatologia de raiva, sendo então sacrificados.

Do total de quirópteros recebidos, foram classificados 257, dos quais 165 pertenciam à família Molossidae (insetívoros), 55 à família Phyllostomidae (frugívoros, nectarívoros), 36 à família Vespertilionidae (insetivoros) e 1 à família Noctillionidae (psívoros). Portanto, os morcegos de hábito alimentar insetívoros foram os recebidos em maior número (201), correspondendo a 78,2\% (Silva e col. ${ }^{15}$ ).

\section{Discussão}

Caso se pretenda manter a raiva urbana sob controle, torna-se essencial esclarecer o papel desempenhado pelo morcego insetívoro na epidemiologia dessa zoonose, visto os casos já descritos na literatura comprovando a presença do vírus rábico nessas espécies.

A adaptação do morcego insetívoro, que constitui a maior parte da população de morcegos ${ }^{10,13,17}$, ao meio urbano se deve em grande parte a oferta abundante de alimento e abrigo das cidades, associada à ausência de predadores. O número desses animais nas áreas urbanas tem se elevado ${ }^{17}$, tendência também observada pelo Servico de Diagnóstico de Morcego do Município de São Paulo.

Outro aspecto importante é a participação do morcego insetívoro no equilíbrio ecológico da população de insetos, uma vez que consomem diariamente grande quantidade dos mesmos. Sobre esse aspecto, Goodwin e Greenhall ${ }^{9}$, afirmaram em 1961 "Certamente teríamos uma superabund ância de insetos em regiões tropicais caso não houvessem agentes controladores do número dos mesmos, dentro de um equilíbrio razoável".

Segundo Greenhall ${ }^{10}$, um morcego pode consumir acima da metade do seu peso, em insetos, em uma noite.Tuttle ${ }^{18}$, em 1979, estimou que 500 morcegos podem facilmente capturar 500.000 insetos em uma noite, portanto não deve ocorrer combate indiscriminado a essas espécies que desempenham papel importante, e talvez indispensável, na eliminação de insetos noturnos.

Nos dois casos positivos identificados no presente estudo, os morcegos foram encontrados, ainda vivos, em locais não habitualmente frequentados por morcegos. Um no chão da sala de um apartamento no $7^{\circ}$ andar de um prédio residencial e outro no muro de uma casa residencial, ambos no Município de São Paulo. Nessas circunstâncias, a possibilidade de ocorrer um acidente envolvendo pessoas, principalmente crianças e animais domésticos é preocupante.

A realização da técnica de IICC com a gordura intersticial tinha por objetivo pesquisar a presença do vírus em uma região potencialmente importan$t^{13}{ }^{13}$, enquanto a inoculação de glândulas salivares objetivava observar a possibilidade de transmissão por via salivar, antes do advento dos sintomas. Os resultados negativos ratificam que os morcegos não são transmissores assintomáticos do vírus rábico como descrito nos primeiros trabalhos ${ }^{5}$ e que outras viroses, que agora são conhecidas como capazes de se alojar em glândulas salivares de morcegos assintomáticos, foram erroneamente identificadas como raiva?

A ampliação do período de observação dos camundongos, de 21 para 42 dias se prende ao relato de Acha e Szyfres ${ }^{2}$, de que em espécies silvestres o período de incubação é mais prolongado e não determinado. Por essa razão, esse laboratório decidiu dobrar o período de observação. O fato dos camundongos não terem apresentado sintomatologia de raiva em 42 dias não descarta a possibilidade, ainda que rara, de que com um período de observação maior venham a apresentar essa sintomatologia.

A observação de que os morcegos insetívoros parecem ser cada vez mais freqüente ${ }^{1} \mathrm{em}$ área urbana e podem estar albergando o vírus rábico, associada a sua importância no equilíbrio ecológico da população de insetos, assim como o pouco conhecimento do seu papel na epidemiologia da raiva em área urbana, deve ser considerada na elaboração de qualquer estratégia de controle da população de morcegos.

ALMEIDA, M. F. et al. [Laboratory diagnosis of rabies in chiroptera carried out of a metropolitan area of South-eastern Brazill. Rev. Saúde Pública, 28: 341-4, 1994.Between January, 1988 and December, 1992 the S. Paulo Animal Disease Control Center subjected 289 bats to rabies examinations, utilizing the direct immunofluorescence and biological techniques. Two insectiverous bats belonging to the species Nyctinomops macrotis, representing $0.69 \%$ of the total sample, tested positive. Both animals had been captured alive in residential neighborhoods of the city, one in 1988 in the living room of a 7'th floor apartment and the other in 1990 on top of a wall surrounding a private house. In the biological tests, the disease showed incubation periods of 13 and 11 days respectively. Although the existence of infected insectiverous bats in urban areas is cause for concern, indiscriminate predatory action against such species can in no way be justified-particularly bearing in mind their importance in preserving the ecological balance of the insect population so prevalent in cities.

\section{Referências Bibliográficas}

1. ACHA, P.N. Epidemiology of paralitic bovine rabies and bat rabies. Bull. Office Int. Epiz., 67:343-82, 1967.

2 ACHA,P.N. \& SZYFRES, B. Zoonosis y enfermedades transmisibles comunes al hombre a los animales. $2^{2}$ ed.Washington, D.C., Organización Panamericana de la 
Salud, 1986. (OPAS-Publicación Científica, 503).

3. BAUER, A.G. \& CRUSIUS, V.A. Isolamento do vírus rábico de morcego insetívoro na cidade de São Leopoldo no Rio Grande do Sul. In: Conferência Anual da Sociedade Veterinária do Rio Grande do Sul, 4. Porto Alegre, 1965. p. 95.

4. CARINE, A. Sur une grande epizootie de rage. Ann. l'Inst. Pasteur. 25:843-6, 1911

5. CONSTANTINE, D.G. Bats in relation to the health welfare and economy of man. In: Wimatt, W. A. Biology of bats. New York, Academic Press, 1970. v.2, p. 319-449.

6. CONSTANTINE, D.G., Un updated list of rabies infected bats in North América. J.Wildl.Dis., 15:347-9, 1979.

7. CONSTANTINE, D.G. Health precautions for bat researchers. In: Kunz, T.H. Ecological and behavioral methods for the study of bats. Washington, Smithsonian Inst. Press, 1988.

8. GARDNER, S.D. Bat rabies in Europe. J. Infect., 18: 205-8, 1989.

9. GOODWIN, G.G. \& GREENHALL, A.M. A review of the bats of a Trinidad and Tobago. Bull Am. Mus. Nat. Hist., 122: 187-302, 1961.

10. GREENHALL, A. M. House bat management. Res. Pub., 143: $1-29,1982$.

11. HAUPT, H. \& REHAAG, H. Raiva epizoótica nos rebanhos de Santa Catarina, sul do Brasil, transmitida por morcegos. Bol. Soc. Bras. Med. Vet., 2:17-47,1925.

12. LARCHI, O. P. Anticuerpos fluorescentes para rabia. Buenos Aires, Centro Panamericano de Zoonosis, 1975. (Nota Técnica, 8, rev. 2).

13. NOWAK, R.M. Order Chiroptera. In: Walker, E. P. Mammals of the world. $3^{\text {rd }}$ ed. Baltimore, Johns Hopkins Univ. Press, 1975.

14. PAWAN, J. L. The transmission of paralitic rabies Trinidad by the vampire bats. Ann. Trop. Med. Parasitol, 30-101-30, 1936.
15. SILVA, M. M. S. et al. Lista das espécies de quirópteros encontrados em municípios de São Paulo. In: Reunião do Instituto Biológico, 6. São Paulo, 1993. Resumos. São Paulo, Instituto Biológico, 1993. Resumo 54.

16. SILVA, R. A. et. al. Isolamento de vírus rábico de morcego não hematófago da espécie Phyllostomos hastatus hastatus. Arq. Inst. Biol. Anim., 4:115-20, 1961.

17. TADDEI, V. A. Morcegos: algumas considerações sistemáticas e biológicas. Bol. Tec. Coord. Assist. Téc. Integral, 172: $1-31,1983$

18. TUTTLE, M.D. Bats-order chiroptera. In; Allen, T. B. \& Scott, S.L. Wild animals of North América. Washington D.C., National Geographic Society,1979. p. 47-76.

19. UIEDA, W. et al. Lista das espécies de morcegos diagnosticadas com raiva no Brasil. In : Seminário Nacional da Raiva, São Paulo, 1992. Resumo 12.

20. UIEDA, W. et al. Raiva em três espécies de molossídeos do Estado de São Paulo. In: Reunião do Instituto Biológico, $5^{\text {a }}$, São Paulo, 1992. Resumo 62.

21. VENTERS, H.D.et al. Rabies in bats in Florida. Am. J. Public Health., 44:182-5, 1954

22. VIZOTTO, L. D. \& TADDEI, V. A. Chave para determinação de quirópteros brasileiros. Rev. Fac. Filos. Cien. Letr. S. José R. Preto Bolm. Cienc., 1:1-72, 1973.

23. WEBSTER, L. T. \& DAWSON, J.R. Early diagnosis of rabies by mouse inoculation. Measurement of humoral immunity to rabies by mouse protection test. Proc. Soc. Exp. Biol. Med., 32: 570-3, 1935.

Recebido para publicação em 29.3.1994 Reapresentado em 1.8 .1994 Aprovado para publicação em 31.10.1994 